\title{
Spatial Outcomes of Myocardial Infarction (Heart Attack) from 2000 to 2018 in Southern West Virginia Using an Collective Analysis.
}

Frank Annie ${ }^{1}$, Mark C Bates ${ }^{2}$, Aravinda Nanjundappa ${ }^{2}$, Ali Farooq ${ }^{2}$, Elise Anderson ${ }^{2}$, and Megan Wood $^{2}$

${ }^{1}$ Affiliation not available

${ }^{2}$ CAMC Vascular Center of Excellence, Area Medical Center, Charleston Area Medical Center, and Charleston Area Medical Center Research Institute and Center for Clinical Sciences Research, CAMC Health Education and Research Institute

November 11, 2020

\section{Author Block}

Frank H Annie, $\mathrm{PhD}^{1}$, Mark C Bates, $\mathrm{MD}^{2}$, Aravinda Nanjundappa, $\mathrm{MD}^{2}$, Ali Farooq, $\mathrm{MD}^{2}$, Elise Anderson, $\mathrm{MD}^{2}$, Megan Wood, $\mathrm{DNP}^{2}$

${ }^{1}$ CAMC Health Education and Research Institute

3200 MacCorkle Ave. SE, Charleston, WV 25304.

${ }^{2}$ CAMC Vascular Center of Excellence, Charleston Area Medical Center.

3200 MacCorkle Ave SE, Charleston, WV 25304

Study Locations:

Charleston Area Medical Center, 3100 McCorkle Ave SE, Charleston, WV, 25302 and Charleston Area Medical Center Research Institute and Center for Clinical Sciences Research, 3200 McCorkle Ave SE, Charleston, WV, 25302

Correspondence:

Frank Annie M.A; MPA, PhD

Research Scientist

CAMC Health Education and Research Institute

3200 MacCorkle Ave. SE,

Charleston, WV 25304

Phone 304-388-9921

Fax: 304-388-9921

Email: Frank.H.Annie@camc.org

Total word count: 750 


\section{Author Disclosure Block: None}

Key words: Spatial Outcomes, MI, West Virginia

Running Title: Spatial Outcomes of Myocardial Infarction (Heart Attack)

Total Number of Tables and Figures: Figures 1

Data concerning the frequency and prevalence of heart disease within the US is not uniform to the entire country. Some regions have experienced increased progress relating to improving access and preventive measures associated with heart disease. Other regions are lagging and continue to have persistent challenges with heart disease. Most literature seeks to understand regions from the county level (1-3). Researchers have used county levels to understand the disease strands associated with Myocardial Infarction and stroke, as well as other disease states. This current work seeks to understand disease states at a zip code level to understand sub-regional differences beyond the county level. Other academic pieces, such as geographical clustering of incidence at a localized level, further explored MI in Denmark at a city neighborhood level to find differing patterns of $\mathrm{MI}$ in that country (2). A larger level of analysis provides a snapshot of differing MI trends than at the local level. Further work, such as that of Ersbool (2015) (4), considered MI at an address level and found different patterns when compared to a larger level, such as city, county, or state. The understanding of different trends at a localized level promotes the field of multi-spaced differences even from neighboring cities.

This is a retrospective analysis of patients admitted or transferred to Charleston Area Medical Center in Charleston, West Virginia. We obtained data from the CAMC data warehouse ranging between 2000 and 2018. We used ICD-9 and ICD-10 codes to identify cases of MI between 2000 and 2018 treated at CAMC. When we identified these cases, we geocoded them using Arch 10.6, with a 99\% accuracy rate. We identified all-cause MI from 2000 to $2018(\mathrm{n}=37,600)$ and mortality from MI $(\mathrm{n}=1,917)$. We used zip codes to define boundaries of analysis within the neighborhoods examined. We obtained zip code data from the West Virginia Geographic Information Systems (GIS) database.

To understand mortality, we performed a collective analysis to understand the differences in mortality based on differing zip codes in the CAMC service area. This analysis considered the incidences of MI divided by the recorded deaths from those zip codes and converted deaths into a percentage and visually represented it in a nested map in Figure 1.To further analyze the incidence of MI and mortality within individual zip codes in Southern West Virginia, we calculated mortality in each individual zip code over the 18-year period of the study. These figures are illustrated in Figure 1, which shows a very high mortality in Southern West Virginia.

In conclusion, incidence and mortality appear to increase in Southern West Virginia. The goal of this project was to define the geographical landscape of MI within Southern West Virginia. The collective analysis also showed that mortality appeared to be increasing outside the urban area of Southern West Virginia. Cardiovascular mortality a leading cause of death, contributing to $30 \%$ of global mortality, and it is a public health concern (5). Spatial analysis plays an important role in identifying areas of increased incidence of CVS hospitalizations, which can provide clues as to causal factors related to the increased burden of disease. Previous studies have shown that CVS disease incidence clusters around areas with markedly low socioeconomic status $(2$,$) . Our study reinforces the findings of previous studies by showing that hot spots$ of cardiovascular disease hospitalization have occurred around rural areas and coal fields in Southern West Virginia.

Figure Legend: Figure 1: Buffer analysis of Hospitals and Rural Health Facilities/ Mortality at the zip code level of MI 2000-2018. 

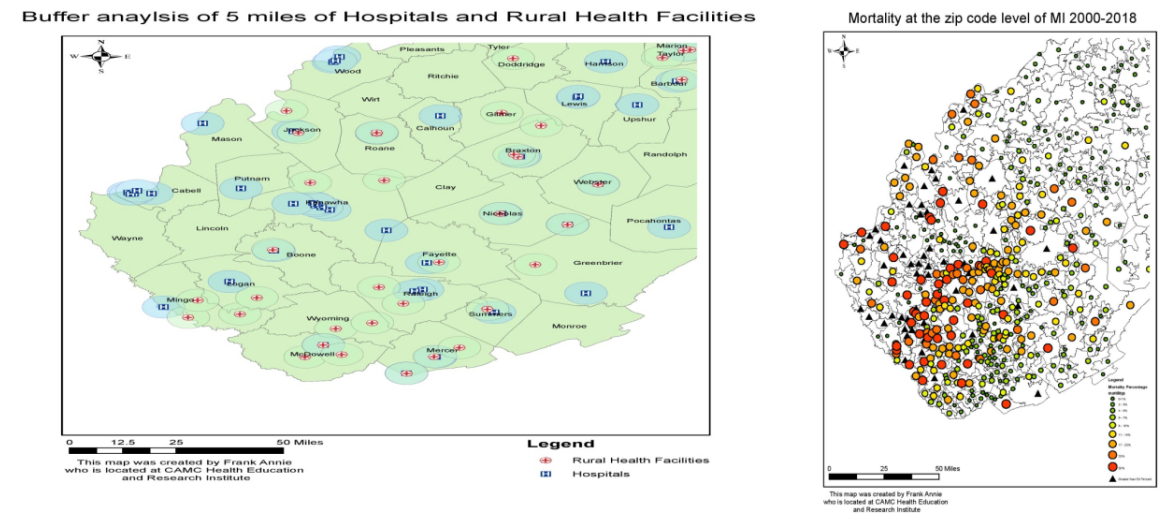

\section{References :}

1. Pedigo, A., \& Aldrich, T. (2011). Neighborhood disparities in stroke and myocardial infarction mortality: a GIS and spatial scan statistics approach. BMC public health , 11 (1), 644.

2. Kjærulff, T. M., Ersbøll, A. K., Gislason, G., \& Schipperijn, J. (2016). Geographical clustering of incident acute myocardial infarction in Denmark: A spatial analysis approach. Spatial and spatiotemporal epidemiology, $19,46-59$.

3. Loughnan, M. E., Nicholls, N., \& Tapper, N. J. (2008). Demographic, seasonal, and spatial differences in acute myocardial infarction admissions to hospital in Melbourne Australia. International journal of health geographics, 7 (1), 42 .

4. Ersbøll, A. K., Kjærulff, T. M., Bihrmann, K., Schipperijn, J., Gislason, G., \& Larsen, M. L. (2016). Geographical variation in a fatal outcome of acute myocardial infarction and association with contact to a general practitioner. Spatial and spatio-temporal epidemiology , 19 , 60-69.

5. Mendis S, Thygesen K, Kuulasmaa K, Giampaoli S, Mahonen M, Ngu Blackett K, et al. World Health Organization definition of myocardial infarction: 2008-09 revision. International journal of epidemiology. 2011;40(1):139-46. 